\title{
Peripheral Pathways Regulate Motoneuron Collateral Dynamics
}

\author{
Richard Redett, ${ }^{2}$ Rajesh Jari, ${ }^{1}$ Thomas Crawford, ${ }^{3}$ You-Gang Chen, ${ }^{5}$ Charles Rohde, ${ }^{4}$ and Thomas M. Brushart ${ }^{1}$ \\ ${ }^{1}$ Department of Orthopedic Surgery, ${ }^{2}$ Division of Plastic Surgery, and ${ }^{3}$ Department of Neurology, Johns Hopkins Medical Institutions, Baltimore, Maryland \\ 21287, ${ }^{4}$ Department of Biostatistics, The Bloomberg School of Public Health, Johns Hopkins University, Baltimore, Maryland 21287, and ${ }^{5}$ Department of \\ Orthopedic Surgery, University of California Los Angeles-Drew Medical School, Los Angeles, California 90059
}

\begin{abstract}
Motor axons regenerating after repair of mixed nerve reinnervate pathways leading to muscle more often than those leading to skin [preferential motor reinnervation (PMR)]. Motoneurons that initially project collaterals to both muscle and skin prune incorrect projections to generate specificity. The number of motor axon collaterals maintained entirely within cutaneous or muscle pathways, however, is unknown. To overcome this shortcoming, dorsal root ganglion excision has been used to allow only motor axons to regenerate after a peripheral lesion. Motor axon number in reinnervated cutaneous and muscle pathways can then be correlated with the number of parent motoneurons determined by retrograde labeling. The number of collaterals per neuron can be calculated for each environment and the relative roles of pathway and end organ assessed by blocking the distal pathways to prevent target reinnervation.

Without sensory competition, PMR develops in two stages: a limited response to muscle nerve and then a robust response to muscle that may involve retrograde signaling to the proximal pathway. Motoneurons maintain more collaterals in cutaneous nerve than in muscle nerve, even without muscle contact. This difference could result either from increased collateral formation in cutaneous nerve or from increased collateral pruning in muscle nerve. In either instance, these findings confirm that muscle and cutaneous pathways have functionally significant identities that can be recognized by motor axons and can regulate their arborization. Decreased arborization in muscle pathways could promote regeneration by focusing neuronal resources on high-yield projections; increased arborization in cutaneous pathways, conversely, would enhance pathfinding abilities.
\end{abstract}

Key words: axon guidance; retrograde labeling; regeneration; dorsal root ganglion; deafferentation; peripheral nerve

\section{Introduction}

Preferential motor reinnervation (PMR) is the tendency for motor axons regenerating in mixed nerve to reinnervate muscle nerve (M) and/or muscle rather than skin (Brushart, 1988). During early regeneration, individual motoneurons often project collaterals to both cutaneous (C) and muscle pathways. Specificity is then generated by pruning collaterals from cutaneous nerve while maintaining those in muscle nerve [the pruning hypothesis (Brushart, 1990)]. In young animals, PMR occurs in response to muscle nerve alone, suggesting that muscle nerve differs from cutaneous nerve in ways that can be recognized by motor axons and that can, in turn, modify their behavior (Brushart, 1993).

Understanding the context of these experiments, the rat femoral nerve model, is crucial to their interpretation (supplemental Fig. 1, available at www.jneurosci.org as supplemental material). Proximally, at the site of nerve transection and repair, cutaneous and muscle Schwann cell tubes intermingle so that both are accessible to regenerating motor axons (Brushart, 1988, 1993; Wit-

Received July 27, 2005; revised Aug. 26, 2005; accepted Aug. 27, 2005.

This work was supported by National Institutes of Health Grant R01 NS034484. We thank Katherine Weaver for artwork and Pamela Talalay for editing this manuscript.

Correspondence should be addressed to Thomas M. Brushart, Johns Hopkins Orthopedics, 601 North Caroline Street, Baltimore, MD 21287. E-mail: tbrusha@jhmi.edu.

DOI:10.1523/JNEUROSCI.3105-05.2005

Copyright $\odot 2005$ Society for Neuroscience $\quad$ 0270-6474/05/259406-07\$15.00/0 zel et al., 2005). Distally, the femoral nerve bifurcates into terminal cutaneous and muscle branches. The specificity of regeneration is assessed by retrograde double labeling of these branches. Regenerating neurons are then scored as projecting correctly to the muscle branch, incorrectly to the cutaneous branch, or simultaneously to both branches [double labeled (D)].

This technique provides limited information about motoneuron collateral formation, only identifying neurons that simultaneously project collaterals to the distal cutaneous and muscle branches (where retrograde tracers are applied) at the moment of tracer application. Collaterals that will not be recognized include those that do not extend far enough distally to be labeled, those that extend distally but are pruned between labeling intervals, and those that ramify within a single branch and transport one tracer to the same neuron. An alternative approach, counting axons in the tributary nerves, is even less revealing. The femoral muscle branch contains many afferent axons, the behavior of which could mask that of regenerating motor axons (Brushart, 1988). In contrast, numerous axons fail to cross the repair site while others arborize extensively (Witzel et al., 2005). Clearly, a combined technique is needed to determine how many collaterals are maintained by each motoneuron in the cutaneous and muscle branches.

In the current model, dorsal root ganglion (DRG) excision ensures that only motoneurons will regenerate after proximal 
femoral nerve repair. Each tributary nerve can then be characterized by the number of myelinated motor axons it contains and by the number of parent motoneurons that produced these axons. The mean number of myelinated collaterals per neuron in that environment can then be calculated. By blocking the distal pathway, the relative contributions of pathway and end organ to collateralization can be determined. Applying these techniques, motoneurons were found to maintain more collaterals in cutaneous nerve than in muscle nerve, regardless of whether muscle was reinnervated. Muscle and cutaneous nerve thus differ in ways that can be recognized by motor axons and that can regulate their arborization. Decreased arborization in muscle pathways could focus resources on axons with a demonstrated potential for success; increased arborization in cutaneous pathways, conversely, would focus continued efforts on pathfinding.

\section{Materials and Methods}

Surgical procedures. Experiments were performed on the femoral nerves of juvenile (3-4 weeks of age; 50-75 g) female Sprague Dawley rats (supplemental Fig. 1, available at www.jneurosci.org as supplemental material) (Brushart, 1988). Juveniles were chosen for these experiments because of previous evidence of pathway recognition in this age group (Brushart, 1993). In the proximal femoral nerve, at the site of transection and repair, axons destined for skin and muscle intermingle. Regenerating axons that contact the distal stump will thus have access to Schwann cell tubes that lead either to muscle or to skin. Distally, the nerve bifurcates into a muscle branch to the quadriceps, containing both motor efferent and muscle afferent fibers and a purely afferent cutaneous branch. Myelinated axon counts are greater in the cutaneous branch (muscle, 1251; cutaneous, 1586), whereas total axoplasmic area is greater in the muscle branch (muscle, 12,263 $\mu \mathrm{m}^{2}$; cutaneous, $7505 \mu \mathrm{m}^{2}$ ), ensuring a balance of factors that might contribute to preferential reinnervation (Brushart, 1988).

Animals were anesthetized by intramuscular injection of ketamine ( 87 $\mathrm{mg} / \mathrm{kg})$ and xylazine $(13 \mathrm{mg} / \mathrm{kg})$. Two types of experimental nerve repair were each assessed at three time periods, resulting in six experimental groups. In "pure motor-end organ" preparations (EO), dorsal root ganglionectomy ensured that only motor axons could reinnervate the distal nerve stump after femoral nerve transection and repair performed under the same anesthetic. In these animals, the distal femoral cutaneous and muscle branches remained connected to their respective end organs. Unilateral laminectomy was performed from L2-L5, and adjacent facet joints were excised with a $1 \mathrm{~mm}$ rongeur to expose the underlying DRGs. The dura mater was incised on the superior aspect of the DRG, and the dorsal root was transected just proximal to the ganglion. The dorsal root stump was then gently elevated, and the ganglion was stripped from underlying ventral root fibers by severing the ganglion capsule with the tip of a 30 gauge needle. The sensory fibers were then transected as they exited the ganglion distally. Ganglia L2-L4 were excised in all preparations; L5 was included when a small L2 contribution to the femoral nerve was noted, a condition found to correlate with an L5 contribution. The ipsilateral femoral nerve was then transected proximal to the iliacus branch with microscissors under 20-40× magnification, and the proximal and distal stumps were sutured within a silicon tube [inner diameter (ID), 0.025 inches; Dow Corning, Midland, MI] to maintain a $0.5 \mathrm{~mm}$ gap between their surfaces. This initially unstructured gap minimizes the effects of intraneural anatomy on the specificity of regeneration (Brushart, 1988). All femoral repairs were re-explored 2 weeks later, the tubes were removed to prevent late compression of the nerve, and animals that evinced disruption of the suture line or a change in the interstump gap were excluded from the study. Three groups of 24 unilateral pure motorend organ rats were prepared, with a goal of evaluating 20 each at 2,3 , and 8 weeks.

Pure motor no-end organ animals (NEO) were prepared to explore the ability of motor axons to respond specifically to isolated muscle and cutaneous pathways by selective reinnervation and/or selective collateral pruning. The experiments described above were repeated, with the addi- tion that the muscle and cutaneous branches were transected at the level of quadriceps innervation and sewn within a blocked-off silicon tube (ID, 0.012 inches; Dow Corning) to prevent end-organ reinnervation. Three groups of 12 animals were prepared with a goal of evaluating 10 each at 2, 3, and 8 weeks.

Retrograde labeling and tissue preparation. At the end of the regeneration interval, the femoral cutaneous and muscle branches were exposed bilaterally. In groups in which pathway-end organ continuity had been maintained, the muscle branch was severed $1 \mathrm{~mm}$ proximal to its penetration of the quadriceps, and the sensory branch was cut at an equivalent distance from the femoral nerve bifurcation to produce proximal cutaneous and muscle stumps of equal length. In groups in which pathwayend organ continuity was interrupted, the muscle and cutaneous branches were removed from their blocked tubes, and the ends were trimmed back $1 \mathrm{~mm}$ to healthy nerve tissue. The distance from the femoral bifurcation at which stumps were exposed to tracer was thus the same in all groups.

One stump (randomly chosen) was immersed in 10\% HRP (Sigma, St. Louis, MO) for $1 \mathrm{~h}$ in a Vaseline (Sherwood, St. Louis, MO) well, after which it was copiously irrigated and loosely sutured to a distant portion of the wound. The other stump was then exposed to 3\% Fluoro-Gold (FG) (Schmued and Fallon, 1986; Brushart, 1990) for 2 h, similarly irrigated, and sewn to the opposite corner of the wound to prevent crosscontamination by diffusion of tracers. Forty-eight hours were allowed for proximal transport of the tracers, after which the animals were deeply anesthetized and perfused through the left ventricle. A warm saline flush $(150 \mathrm{ml})$ was followed by $500 \mathrm{ml}$ of $4 \%$ paraformaldehyde in $0.1 \mathrm{M}$ sodium acetate buffer, $\mathrm{pH} 6.5$, over one-half hour, $500 \mathrm{ml}$ of $4 \%$ paraformaldehyde in $0.1 \mathrm{~m}$ sodium borate buffer, $\mathrm{pH} 9.5$, over one-half hour, and $500 \mathrm{ml}$ of $10 \%$ sucrose in $0.1 \mathrm{M}$ Sorensen's phosphate buffer, $\mathrm{pH} 7.4$, over one-half hour (Berod et al., 1981; Mesulam, 1982). The lumbosacral spinal cords were removed, and serial cross-sections were cut at $40 \mu \mathrm{m}$ on a freezing microtome. The ventral roots remaining at the previous DRG sites were also removed, embedded in albumin-gelatin, and sectioned longitudinally at $40 \mu \mathrm{m}$ to evaluate the completeness of ganglion excision. All sections were reacted with $\mathrm{H}_{2} \mathrm{O}_{2}$ and tetramethyl benzidine to demonstrate HRP within neurons (Mesulam, 1982). Sections were serially mounted on glass slides, dried, and overlaid with coverslips without counterstain to minimize background fluorescence.

The peripheral nerve from all 8-week-old animals was processed for counting myelinated axons. Segments of each femoral cutaneous and muscle branch were postfixed in one-fourth strength Karnovsky's fixative for $72 \mathrm{~h}$, stored in $0.1 \mathrm{~m}$ phosphate buffer, and then embedded in Epon-Araldite. One-micrometer cross sections of each cutaneous and muscle branch were cut, counterstained with toluidine blue, and mounted on glass slides.

Evaluation of motoneuron labeling. Each $40 \mu \mathrm{m}$ spinal cord section was viewed with fluorescent $(405 \mathrm{~nm})$ and transmitted light at $20-40 \times$ by an observer unaware of which tracer had been used on which femoral branch. Counts were prepared for each nerve of HRP-labeled motoneurons, FG-labeled motoneurons, and double-labeled motoneurons. The presence of split cells in adjacent sections was corrected as described by Abercrombie (1946). Each of the six experimental groups was then characterized by three means: the mean number of motoneurons projecting correctly to the muscle branch, the mean number projecting incorrectly to the cutaneous branch, and the mean number of double-labeled neurons, those projecting axon collaterals to both branches. Multiple regression analyses were used to compare means of correct, incorrect, and double-labeled neuron counts to one another both within and among each of the six groups.

Axon counting. Cross sections of cutaneous and muscle nerve from 8 -week-old end organ and no-end organ groups were examined at $1370 \times$ by using a Nikon (Tokyo, Japan) Optiphot microscope interfaced with Bioquant software (R\&M Biometrics, Nashville, TN). Myelinated axons were counted by standard stereologic methods (Mayhew, 1988). Each nerve was characterized by the total number of myelinated fibers regenerating and the mean diameter of these fibers. A Poisson regression analysis was used to compare the number of axons per motoneuron in the cutaneous and muscle branches at 8 weeks with and without end 
organ. The 8 week time period was chosen for analysis because nearly all regenerating motor sprouts are myelinated by this time (T. M. Brushart, unpublished data) and can be quantified by light microscopy. Furthermore, limiting the study to myelinated axons includes all motor collaterals that have matured to the point of functional competence, regardless of their destination.

Control experiments. Control experiments were performed to validate the pure motor model. At issue are the damage caused to motor axons by DRG excision and the ability to correlate labeled motoneuron counts with the number of myelinated axons remaining in the muscle and cutaneous branches. The number of motoneurons projecting to the muscle and cutaneous branches of untransected femoral nerves was determined with $\mathrm{HRP} / \mathrm{FG}$ double labeling in normal animals $(n=6)$, both 1 week $(n=5)$ and 8 weeks $(n=5)$ after generating pure motor nerve by excising the L2-L4 DRGs. These time periods were picked to assess the immediate effects of ganglion excision, as soon as Wallerian degeneration has cleared the denervated sensory Schwann cell tubes, and the later consequences, when sprouts from injured motor axons have had an opportunity to regenerate distally. Femoral cutaneous and muscle branches from the 1 week pure motor control group were embedded in plastic, sectioned at 1 $\mu \mathrm{m}$, and evaluated to determine the number and size of remaining myelinated axons.

\section{Results}

\section{Motoneuron counts}

In the pure motor-end organ groups (EO), preferential motor reinnervation was already present at 2 weeks, with significantly more motoneurons projecting correctly to muscle than incorrectly to skin [M, 126 (SE 13); C, 57 (SE 8); $p<0.0001$ ] (Fig. 1). The number of correct projections increased dramatically during each subsequent time interval [EO-M, 2 weeks, 126, EO-M, 3 weeks, 194 (SE 13), $p<0.001$; EO-M, 3 weeks, 194, EO-M, 8 weeks, 279 (SE 16), $p<0.0001$ ]. During this process, the number of motoneurons projecting incorrectly to skin increased between 2 and 3 weeks [EO-C, 2 weeks, 57 (SE 8); EO-C, 3 weeks, 91 (SE 9); $p=0.003$ ] but then leveled off at a value not significantly different from the means at 2 or 3 weeks [EO-C, 8 weeks, 76 (SE 7 ); $p=0.100$ (vs 2 weeks) and $p=0.165$ (vs 3 weeks)]. By 8 weeks after nerve repair, more than three and one-half times as many motoneurons projected correctly to the muscle branch as projected incorrectly to the cutaneous branch. The number of double-labeled motoneurons, those maintaining projections to both muscle and cutaneous branches, did not change significantly throughout the experiment [EO-D, 2 weeks, 31 (SE 4), EO-D, 3 weeks, 33 (SE 5), $p=0.650$; EO-D, 3 weeks, 33, EO-D, 8 weeks, 25 (SE 3); $p=0.310$ ].

The pure motor no-end organ animals were strikingly similar to their counterparts with end-organ access 2 weeks after nerve repair. The mean number of motoneurons projecting correctly to muscle [EO-M, 2 weeks, 126; NEO-M, 2 weeks, 125 (SE 11); $p=$ 0.959] and incorrectly to skin [EO-C, 2 weeks, 57; NEO-C, 2 weeks, 56 (SE 6); $p=0.884]$ were virtually identical. Preferential motor reinnervation may thus be expressed at 2 weeks in response to the muscle pathway, without need for end-organ contact. Between 2 and 8 weeks, however, additional specific regen- eration does not occur without end-organ participation. There was no significant increase in the number of correct projections to muscle between 2 and 3 weeks [NEO-M, 2 weeks, 125; NEO-M, 3 weeks, 141 (SE 19); $p=0.427$ ] or between 3 and 8 weeks [NEO-M, 3 weeks, 141; NEO-M, 8 weeks, 155 (SE 8); $p=$ 0.113 ]. Interestingly, the mean number of incorrect projections to the skin was not altered by the presence or absence of the end organ at any time period [EO-C, 2 weeks, 57, NEO-C, 2 weeks, 56 (SE 6), $p=0.884$; EO-C, 3 weeks, 91, NEO-C, 3 weeks, 76 (SE 14), $p=0.336$; EO-C, 8 weeks, 76, NEO-C, 8 weeks, 70 (SE 11), $p=$ $0.624]$. The nature of an incorrect projection can thus be determined by the pathway alone. The number of motoneurons maintaining dual projections to muscle and cutaneous nerve was also modified by end-organ contact. More motoneurons were double labeled if end-organ access was denied at both 2 weeks [EO-D, 2 weeks, 31; NEO-D, 2 weeks, 50 (SE 10); $p=0.042$ ] and 3 weeks [EO-D, 3 weeks, 33; NEO-D, 3 weeks, 74 (SE 12); $p=0.001$ ], suggesting that collateral pruning is stimulated by contact with muscle. The final value at 8 weeks, however, was not end-organ dependent (EO-D, 8 weeks, 25; NEO-D, 8 weeks, 33; $p=0.177$ ).

\section{Axon counts}

Myelinated motor axon counts were obtained from the muscle and cutaneous branches of EO and NEO animals 8 weeks after ganglion excision and nerve repair. When end-organ contact was permitted, a mean of 818 (SE 56) myelinated axons reinnervated the muscle branch, and a mean of 513 (SE 35) reinnervated the cutaneous branch $(p<0.0001)$. If end-organ contact was denied, a mean of 560 (SE 58) axons reinnervated the muscle branch, and a mean of 501 (SE 95) reinnervated the cutaneous branch $(p=0.6004)$. Comparing EO and NEO groups, endorgan contact significantly influenced axon counts in the muscle 


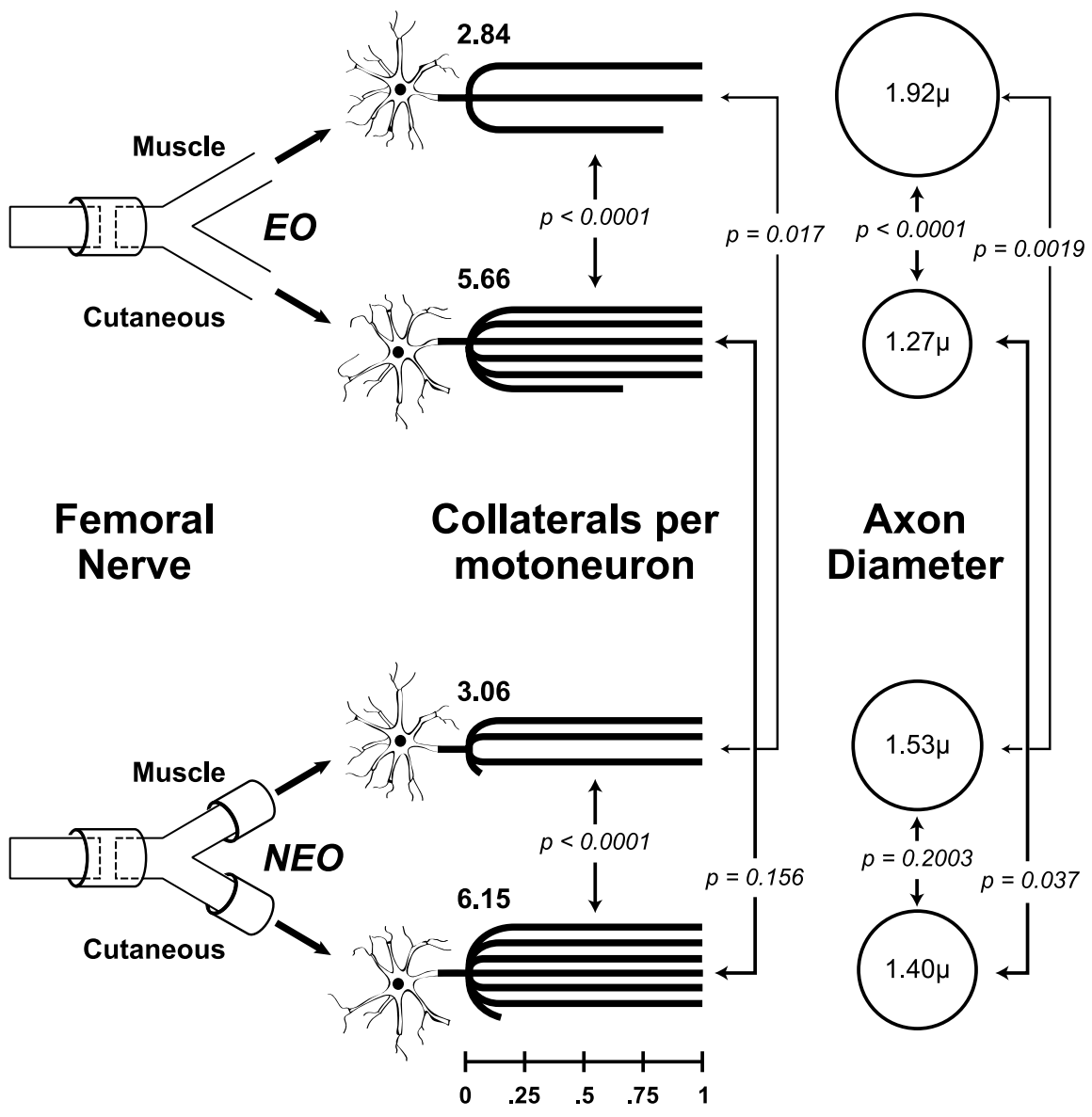

Figure 2. The number of collaterals per motoneuron and their diameters 8 weeks after femoral nerve repair. Significantly more collaterals per neuron are maintained in cutaneous nerve than in motor nerve, and their number is independent of end-organ contact. In contrast, motoneurons that project to muscle nerve maintain significantly more collaterals if muscle contact is denied. Increased collateralization thus appears to reflect negatively on the axonal environment. Axon diameter is a less-sensitive measure of environment, differing significantly between cutaneous and muscle nerve only when muscle reinnervation is permitted.

persisting in the cutaneous branch $(p=$ 0.156). In aggregate, these results show that motoneurons maintain fewer collaterals in the muscle branch if end-organ contact is permitted but respond to the cutaneous branch regardless of end-organ availability. Additionally, motoneurons are clearly able to respond to pathway identity alone, because the muscle branch contained only half as many collaterals as the cutaneous branch even if these collaterals were unable to interact with muscle.

The mean diameter of myelinated motor axons did not differ significantly between cutaneous and muscle nerve in $\mathrm{NEO}$ animals but increased significantly in the muscle nerve when it led to muscle (Fig. 2).

\section{Control experiments}

In normal animals $(n=6)$, a mean of 347 (SE 14) motoneurons projected to the muscle branch, and none projected to the cutaneous branch. One week after DRG excision without nerve repair $(n=10)$, a mean of 369 (SE 19) motoneurons projected to the muscle branch, and none projected to the cutaneous branch. DRG excision thus had no significant effect on baseline motoneuron projections ( $p=$ $0.60)$. Eight weeks after DRG excision without nerve repair $(n=5)$, however, a mean of 402 (SE 24) motoneurons projected to the muscle branch, a mean of 74 (SE 7) projected to the cutaneous branch, and a mean of 27 (SE 9) were doublelabeled, significant increases in all categories $(p<0.01)$. Many of these additional

branch $(p=0.0071)$ but not in the cutaneous branch $(p=$ 0.8829).

The outcome of these experiments can be better appreciated by determining the number of myelinated axon collaterals per motoneuron in the cutaneous and muscle branches of EO and NEO animals. For these calculations, the total motoneuron count for each branch is taken to be the number of motoneurons projecting only to that branch plus the number of double-labeled motoneurons. Although double-labeled motoneurons may have fewer collateral projections than these calculations assume, their relative numbers are small ( $7 \%$ of labeled motoneurons), so that any resulting inaccuracy will have little effect on the results. The total number of myelinated axons in each branch is then divided by the total motoneuron count labeled from that branch to determine the number of collaterals per motoneuron.

When end-organ contact was permitted, motoneurons maintained a mean of 5.66 (SE 0.41) collaterals in the cutaneous branch and 2.84 (SE 0.16) collaterals in the muscle branch $(p<$ 0.0001 ) (Fig. 2). If end-organ contact was denied, a mean of 6.15 (SE 0.66) collaterals was maintained in the cutaneous branch, whereas a mean of 3.06 (SE 0.36) persisted in the muscle branch $(p<0.0001)$. Comparing EO and NEO groups, end-organ contact significantly influenced the number of myelinated collaterals maintained by each motoneuron in the muscle branch $(p=$ 0.017 ) but had no significant effect on the number of collaterals motoneurons lay outside the anatomical confines of the normal femoral motoneuron pool (Fig. 3), suggesting innervation of the femoral nerve by nonfemoral axons as a result of DRG excision. Evaluation of peripheral nerve from pure motor animals 1 week after surgery $(n=5)$ revealed a mean of 356 (SE 21) myelinated axons in the muscle branch and none within the cutaneous branch (Fig. 4). This number compares favorably with the mean of 369 motoneurons labeled from these same branches (see above), indicating a nearly one-to-one correspondence between motoneuron labeling and myelinated axon counts within the same nerve. Ventral root remaining at the site of DRG excision contained no labeled sensory neurons, consistent with effective deafferentation.

\section{Discussion}

The modified femoral nerve model

DRG excision to remove afferent axons followed by HRP labeling of motoneurons was introduced by Peyronnard et al. (1986) to evaluate labeling efficiency. When DRG excision is added to the femoral nerve model, only motor axons will regenerate after nerve repair. We are thus able to correlate, for the first time, the number of motor axon collaterals in a peripheral pathway with the number of motoneurons that generated these collaterals. Although deafferentation is known to compromise motoneuron development (Kalb and Hockfield, 1992) and function (Mendell 

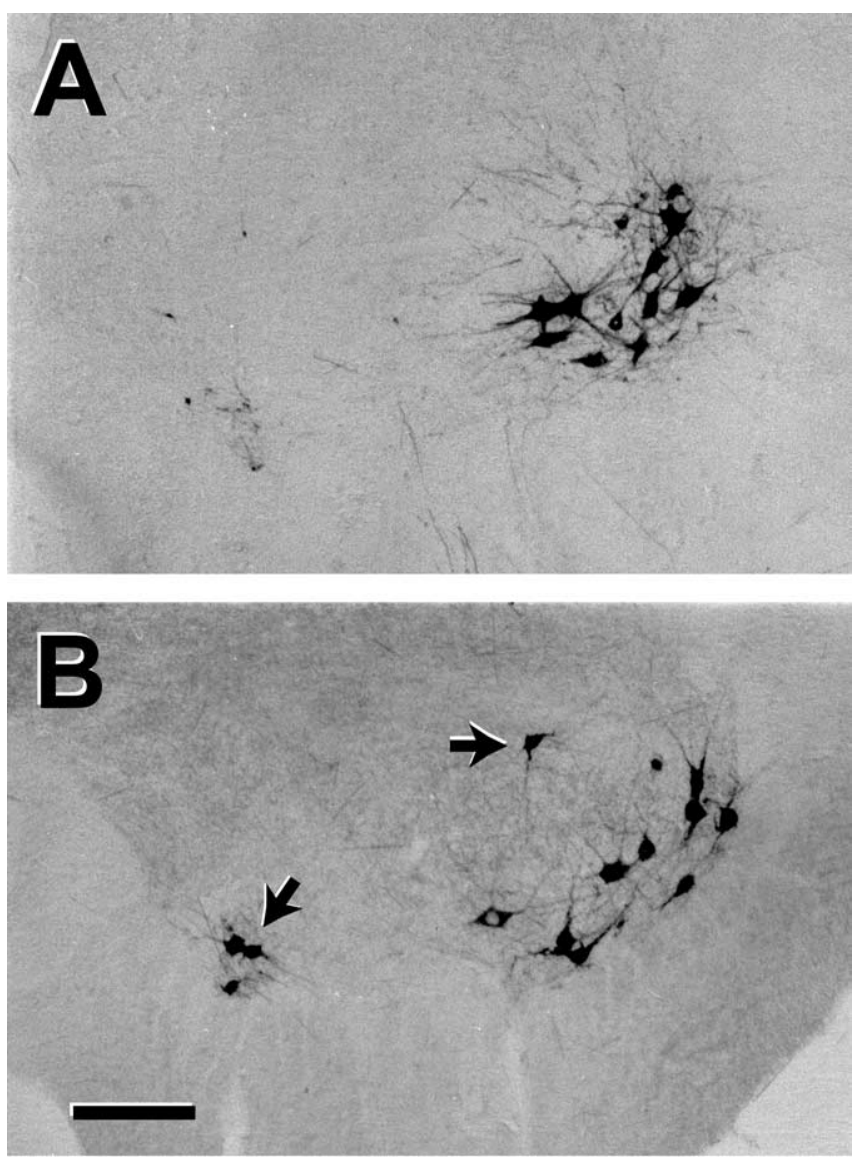

Figure 3. Motoneuron labeling from the femoral muscle branch in normal rats $(\boldsymbol{A})$ and 8 weeks after femoral nerve repair and excision of the $L 2, L 3$, and $L 4 D R G S(B)$. The femoral nerve is normally innervated by a discrete pool of motoneurons. After ganglion excision, some motoneurons are recruited from distant motoneuron pools, presumably as a result of sprouting into pathways vacated by sensory axon loss. Scale bar, $100 \mu \mathrm{m}$.

et al., 1999), it may also have a positive impact on regeneration through upregulation of BDNF in the neuron (Johnson et al., 2000).

Control experiments were necessary to confirm successful removal of afferent axons and to evaluate possible damage to remaining motor axons. One week after deafferentation, myelinated axons were no longer present in the femoral cutaneous branch, whereas areas of degeneration resulted from loss of smaller myelinated afferents in the muscle branch (Fig. 4). The mean number of motoneurons labeled from the deafferented muscle branch at this time (369) was similar to the number labeled in normal animals (347), confirming that DRG excision can be performed without significant loss of motor axons. Ventral root remaining at the site of DRG excision contained no labeled sensory neurons, consistent with effective deafferentation.

The mean number of motoneurons projecting to the muscle branch after deafferentation increased from 369 at 1 week to 402 at 8 weeks. Ventral roots may be stimulated to sprout by the injury of DRG excision, a process no doubt influenced by adjacent degenerating pathways. These suspicions are confirmed by the location of labeled motoneurons beyond the confines of the normal femoral pool, indicating that they originally projected to other peripheral nerves (Fig. 3). Although this sprouting would defeat attempts to quantify normal anatomy, it does not detract from the current use of the femoral model, as only motor axons are permitted to regenerate. The addition of these neurons ex-
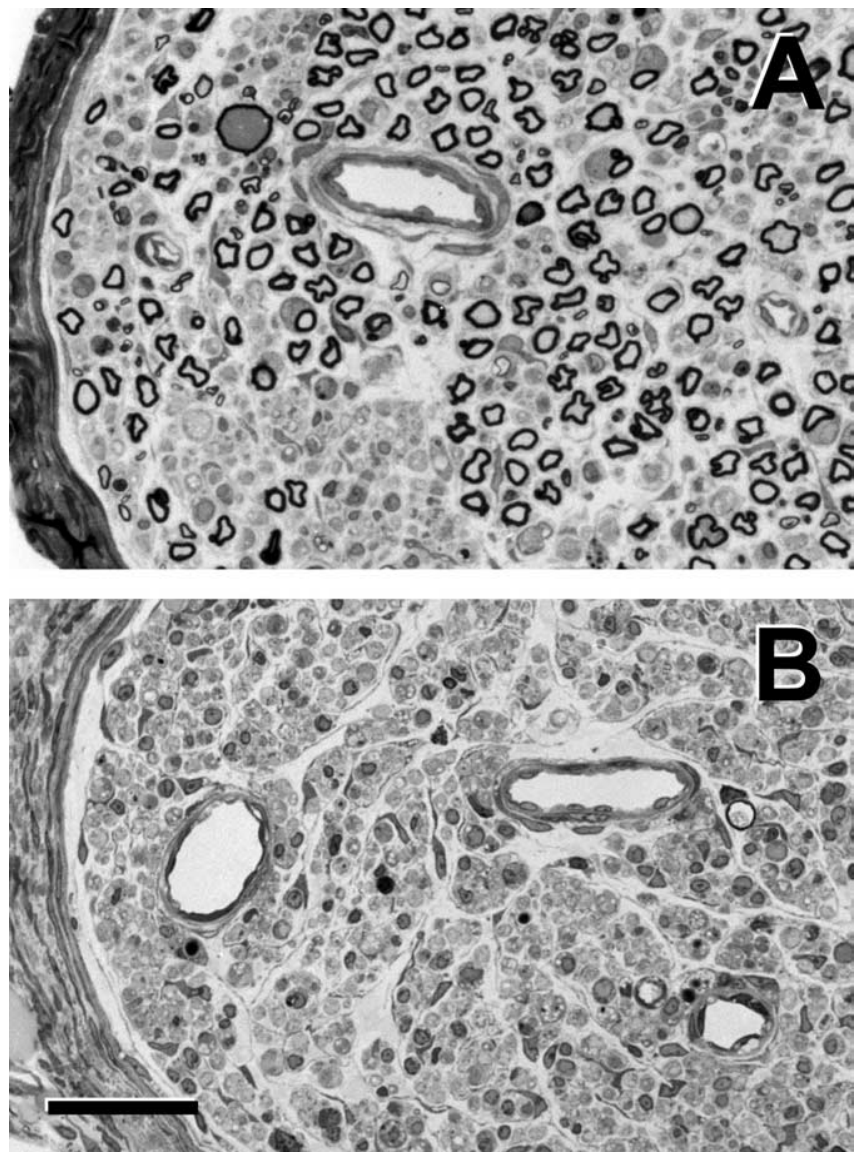

Figure 4. Photomicrographs of the femoral muscle branch $(\boldsymbol{A})$ and cutaneous branch $(\boldsymbol{B}) 1$ week after excision of the L2, L3, and L4 DRGs. Large myelinated axons remain within the muscle branch; areas of dropout were previously occupied by smaller myelinated and unmyelinated axons. No myelinated axons remain within the cutaneous branch. Scale bar, $50 \mu \mathrm{m}$.

plains the increased total regeneration compared with previous experiments in which DRGs were not excised (Brushart, 1993). An increase, rather than a decrease, of the number of labeled motoneurons also indicates that deafferentation has not interfered with motoneuron labeling, a possibility suggested by Peyronnard and Charron (1983).

\section{Preferential motor reinnervation}

These experiments demonstrate that PMR develops in two distinct phases (Fig. 1). Two weeks after nerve repair in EO animals, twice as many motoneurons project to the muscle branch as to the cutaneous, and few are double labeled. A nearly identical pattern is seen in NEO animals, confirming that the initial phase of PMR results from axon/pathway interaction without reference to muscle. The course of subsequent regeneration is determined by end-organ availability. If muscle contact is permitted, the number of correct projections increases dramatically. If muscle contact is denied, however, there is no significant change in correct projections between 2 and 3 weeks, or between 3 and 8 weeks. Muscle contact is therefore a prerequisite for the second phase of PMR. In previous experiments in which cutaneous and motor axons regenerated together (Brushart, 1993), the more prolonged development of PMR masked this now clear distinction between pathway- and muscle-dependent phases.

The pattern of change in the pool of double-labeled motoneurons, those with simultaneous projections to distal cutaneous and muscle nerve at the time of labeling, was influenced by the pres- 
ence or absence of both regenerating sensory axons and end organ. The pruning hypothesis was originally suggested by the pattern after simple nerve repair (Brushart, 1993): a large population of double-labeled neurons at 2 weeks, followed by a reciprocal decrease in double labeling and increase in the number of correct projections. Without sensory competition (EO), the doublelabeled pool remains small throughout the experiment. When denial of end-organ contact is superimposed on this situation, however (NEO), the pool is significantly enlarged at both 2 and 3 weeks. These observations suggest that the number of motoneurons double labeled at a given time is an index of pathfinding difficulty, with ease of pathfinding enhanced by removal of competition from sensory axons, but compromised by lack of interaction with muscle.

The mechanism of PMR will be clarified substantially when we define the turnover of double-labeled motoneurons. One possibility is that all specificity is generated through pruning, with rapid turnover of the double-labeled pool. If it was possible to label continuously, the total number of correct projections would be the sum of randomly correct projections and double-labeled neurons. With this extreme fluidity, the neurons double-labeled in the current experiments would be completely different at each time interval, and many would escape double labeling altogether by extending and pruning their collaterals between labeling intervals. An alternative possibility is suggested by the patterns of specificity generation in EO and NEO groups. The early, pathway-dependent phase of PMR is most likely the result of pruning (Brushart, 1990), yet this mechanism is unable to further enhance specificity between 2 and 8 weeks in NEO animals. When muscle contact is permitted, the double-labeled pool remains small. If the pool is also static, with the same neurons labeled at each interval, then all new projections at 3 and 8 weeks are immediately correct. This observation, coupled with the finding that total regeneration is also enhanced by muscle contact, suggests that interaction with muscle by pioneering motoneurons stimulates retrograde signaling to proximal pathway or motoneuron to promote subsequent regeneration. The idea that target reinnervation by a few axons can modify the course of subsequent peripheral regeneration is consistent with the identification of time to initial target reinnervation as the primary determinant of outcome after primate nerve repair (Krarup et al., 2002).

\section{Collateral generation and pruning}

In the EO group, motoneurons that project correctly to muscle maintain significantly fewer myelinated collaterals than do those that project incorrectly to skin (2.84 vs 5.66 ; $p<0.0001)$, and these collaterals are of larger caliber (1.92 vs $1.27 \mu \mathrm{m} ; p<$ $0.0001)$. This apparent pruning and maturation in response to muscle contact is consistent with the results of early regeneration experiments (Sanders and Young, 1946; Aitken et al., 1947). Axon counts distal to transection and repair of predominantly motor nerve have been found to be elevated by a factor of three to five, depending on the model and postoperative time (Shawe, 1955; Evans and Murray, 1956; Jenq and Coggeshall, 1984). Although compromised by the failure to identify proximal axons that do not cross the repair (Kuypers et al., 1998; Witzel et al., 2005), and by the large number of afferent axons in so-called "motor" nerves (Boyd and Davey, 1968), these estimates are consistent with our current determinations.

The preponderance of collaterals in the cutaneous nerves of $\mathrm{EO}$ animals is an apparent contradiction to the pruning hypothesis, as one might expect reduced collateral numbers after prun- ing has occurred. The pruning that generates PMR is essentially completed by 8 weeks, however, and acts only on motoneurons that project to both cutaneous and muscle nerves. The vast majority of myelinated axons counted are thus collaterals of neurons that project either entirely to cutaneous or muscle nerve but not to both. Because increased axon counts in cutaneous nerve are actually the harbinger of regeneration failure in this setting, they cannot be taken to indicate preferential reinnervation as proposed by Maki (1991).

In NEO animals, motoneurons that cannot interact with muscle support more collaterals than do their counterparts in the EO group. Muscle contact thus stimulates collateral pruning. Even so, the difference in collateral number between EO and NEO muscle nerves is far less than that separating cutaneous and muscle nerve in both groups (Fig. 2). Eight weeks after repair, cutaneous and muscle nerve thus regulate collateralization far more differently than do muscle and muscle nerve, further bolstering the concept of pathway identity. When individual motoneurons project collaterals to different environments, they are able to prune most "incorrect" projections within 8 weeks. Studies of pruning at the population level, in contrast, suggest that most pruning takes place far more slowly (Shawe, 1955; Evans and Murray, 1956; Jenq and Coggeshall, 1984; Mackinnon et al., 1991). One would thus expect to find progressively fewer collaterals in the EO muscle branch after longer postoperative intervals.

The number of collaterals in a given environment must reflect the combined effects of collateral generation and pruning. In NEO animals, the muscle pathway may be conducive to collateral pruning and/or the cutaneous pathway conducive to collateral generation. Pruning in muscle pathways could result from the selective elaboration of factors that partially substitute for muscle-derived support such as the HNK-1 carbohydrate. Normally found in muscle nerve but not in cutaneous nerve, HNK-1 is re-expressed only when motor axons reinnervate previously motor Schwann cell tubes (Martini et al., 1992, 1994). Alternatively, factors limited to cutaneous nerve may selectively promote collateral formation. The expression of both NGF and BDNF is upregulated by a factor of 30 in denervated cutaneous nerve but barely at all in denervated ventral root (Hoke et al., 2004). These factors not only promote sprouting from sensory (Diamond et al., 1992; Gallo and Letourneau, 1998) and retinal ganglion (Cohen-Corey and Fraser, 1995; Lom and Cohen-Corey, 1999) neurons, but their antibodies reduce sprouting from motoneurons (Streppel et al., 2002). The substantial increase in collateralization within cutaneous nerve may thus result from increased sprouting rather than decreased pruning, an adaptation that could promote eventual pathfinding success.

\section{References}

Abercrombie M (1946) Estimation of nuclear population from microtome sections. Anat Rec 94:239-247.

Aitken JT, Sharman M, Young JZ (1947) Maturation of regenerating nerve fibers with various peripheral connexions. J Anat 81:1-22.

Berod A, Hartman BK, Pujol JF (1981) Importance of fixation in immunohistochemistry: use of formaldehyde solutions at variable $\mathrm{pH}$ for the localization of tyrosine hydroxylase. J Histochem Cytochem 29:844-850.

Boyd IA, Davey MR (1968) Composition of peripheral nerves. Edinburgh: Livingston.

Brushart TM (1988) Preferential reinnervation of motor nerves by regenerating motor axons. J Neurosci 8:1026-1031.

Brushart TM (1990) Preferential motor reinnervation: a sequential doublelabeling study. Restor Neurol Neurosci 1:281-287.

Brushart TM (1993) Motor axons preferentially reinervate motor pathways. J Neurosci 13:2730-2738. 
Cohen-Corey S, Fraser SE (1995) Effects of brain-derived neurotrophic factor on optic axon branching and remodeling in vivo. Nature 378:192-196.

Diamond J, Holmes M, Coughlin M (1992) Endogenous NGF and nerve impulses regulate the collateral sprouting of sensory axons in the skin of the adult rat. J Neurosci 12:1454-1466.

Evans DH, Murray JG (1956) A study of regeneration in a motor nerve with a unimodal fiber diameter distribution. Anat Rec 126:311-329.

Gallo G, Letourneau PC (1998) Localized sources of neurotrophins initiate axon collateral sprouting. J Neurosci 18:5403-5414.

Hoke A, Zhou C, Redett R, Jari J, Brushart T (2004) Differential upregulation of neurotrophic factors during reinnervation of sensory and motor nerve grafts. Soc Neurosci Abstr 30:373.2.

Jenq C-B, Coggeshall RE (1984) Regeneration of axons in tributary nerves. Brain Res 310:107-121.

Johnson RA, Okragly AJ, Haak-Frendscho M, Mitchell GS (2000) Cervical dorsal rhizotomy increases brain-derived neurotrophic factor and neurotrophin-3 expression in the ventral spinal cord. J Neurosci 20:RC77(1-5).

Kalb RG, Hockfield S (1992) Activity-dependent development of spinal cord motor neurons. Brain Res Brain Res Rev 17:283-289.

Krarup C, Archibald SJ, Madison RD (2002) Factors that influence peripheral nerve regeneration: an electrophysiological study of the monkey median nerve. Ann Neurol 51:69-81.

Kuypers PDL, Van Egeraat JM, Heel MDV, Van Briemen LJ, Godschalk M, Hovius SER (1998) A magnetic evaluation of peripheral nerve regeneration: I. The discrepancy between magnetic and histologic data from the proximal segment. Muscle Nerve 21:739-749.

Lom B, Cohen-Corey S (1999) Brain-derived neurotrophic factor differentially regulates retinal ganglion cell dendritic and axonal arborization in vivo. J Neurosci 19:9928-9938.

Mackinnon S, Dellon L, O’Brien J (1991) Changes in nerve fiber numbers distal to a nerve repair in the rat sciatic nerve model. Muscle Nerve 14:1116-1122.
Maki Y (1991) Experimental study of selective motor and sensory nerve regeneration (part 4). J Jpn Soc Surg Hand 8:16-19.

Martini R, Xin Y, Schmitz B, Schachner M (1992) The L2/HNK-1 carbohydrate epitope is involved in the preferential outgrowth of motor neurons on ventral roots and motor nerves. Eur J Neurosci 4:628-639.

Martini R, Schachner M, Brushart TM (1994) The L2/HNK-1 carbohydrate is preferentially expressed by previously motor axon-associated Schwann cells in reinnervated peripheral nerves. J Neurosci 14:7180-7191.

Mayhew TM (1988) An efficient sampling scheme for estimating fiber number from nerve cross sections: the fractionator. J Anat 157:127-134.

Mendell LM, Johnson R, Munson JB (1999) Neurotrophin modulation of the monosynaptic reflex after peripheral nerve transection. J Neurosci 19:3162-3170.

Mesulam M-M (1982) Tracing neural connections with horseradish peroxidase, pp 70-104. New York: Wiley.

Peyronnard J-M, Charron L (1983) Decreased horseradish peroxidase labeling in deafferented spinal motoneurons of the rat. Brain Res 275:203-214.

Peyronnard J-M, Charron L, Lavoie J, Messier J-P (1986) Motor, sympathetic and sensory innervation of rat skeletal muscles. Brain Res 373:288-302.

Sanders FK, Young JZ (1946) The influence of peripheral connection on the diameter of regenerating nerve fibers. J Exp Biol 22:203-212.

Schmued LC, Fallon J (1986) Fluoro-gold, a new fluorescent retrograde axonal tracer with numerous unique properties. Brain Res 377:147-154.

Shawe GDH (1955) On the number of branches formed by regenerating nerve-fibers. Br J Surg 42:474-488.

Streppel M, Azzolin N, Dohm S, Guntinas-Lichius O, Haas C, Grothe C, Wevers A, Neiss W, Angelov DN (2002) Focal application of neutralizing antibodies to soluble neurotrophic factors reduces collateral axonal branching after peripheral nerve lesion. Eur J Neurosci 15:1327-1342.

Witzel C, Rohde C, Brushart TM (2005) Pathway sampling by regenerating peripheral axons. J Comp Neurol 485:183-190. 